\title{
Cardiovascular risks of climate change
}

Annette Peters $\mathbb{D}^{1,2,3,4} \bowtie$ and Alexandra Schneider ${ }^{1}$

Extreme heat events are now more frequent in many parts of the world as a result of climate change. The combined effects of heat, air pollution, individual age, and socioeconomic and health status are responsible for avoidable acute events of cardiovascular disease and need to be considered in order to prevent and treat cardiovascular diseases effectively.

Climate change is altering the Earth's global land and ocean temperatures. A $1{ }^{\circ} \mathrm{C}$ increase in global mean surface temperature was observed during the last years of the $2010 \mathrm{~s}$, relative to the pre-industrial period ${ }^{1}$. As a consequence, temperature variability and extreme heat events have become more frequent in many parts of the world. For example, unusually hot weather was observed during the 2003 heatwave in Central Europe. Overall, $>70,000$ deaths were estimated to be attributable to these extreme conditions, with more than one-third of the deaths occurring in France, Italy and Spain ${ }^{2}$. Since then, population exposure to extreme heat has increased worldwide, resulting in an additional 220 million heatwave exposure events in 2018 compared with the average in the years $1986-2005^{3}$.

Analyses of daily death rates have shown that both low and high temperatures are associated with increases in cardiovascular disease mortality ${ }^{3}$. Although this general overall pattern is consistently observed around the world, the regional climate and the cultural and technical adaptation to cold and heat influence the temperature at which the fewest deaths occur. Non-optimal temperature was newly included into the Global Burden of Disease estimation and accounted for 1.96 million deaths globally, with (so far) a larger burden coming from low than from high temperatures ${ }^{4}$. Under the current situation of climate change, the relative importance of extreme weather is changing. Indeed, we were able to observe changes in the heat-related risk of myocardial infarction even in Augsburg, Germany ${ }^{5}$. On the basis of data from the KORA Myocardial Infarction Registry, we assessed the effect of cold and heat on the onset of myocardial infarction in two time periods: $1987-2000$ and 2001-2014. During the first period, myocardial infarctions were triggered by cold exposure only. However, during the second period, we observed significant effects of temperatures $>18^{\circ} \mathrm{C}$ on the risk of myocardial infarction. The increased risk was attributable to the greater vulnerability to cardiovascular disease of individuals with type 2 diabetes mellitus or hypertension. This observation indicates that the health effects of altered temperature exposure are already detectable now and confer an additional burden on vulnerable subgroups of the general population. In the future, we predict that the effects of heat exposure on health will outweigh the reduced effects of cold exposure on health and will produce a substantial burden of non-ST-segment elevation myocardial infarctions under the uncontrolled climate change scenarios that are currently the most likely ${ }^{6}$.

An increase in the risk of acute-onset ischaemic heart disease with increasing temperatures is biologically plausible, given the pathophysiological implications of increased heat exposure. Thermal stress results in physiological responses to changes in central body temperature, leading to increased sweating, elevated heart and breathing rates, vasodilatation, and increased or decreased coagulation. These changes can cause imbalances in the autonomic control of the heart, increase local arterial pressures, induce systemic inflammation and impair clotting responses ${ }^{7}$. Jointly, these disruptions of haemostasis potentially predispose vulnerable individuals to atherosclerotic plaque rupture and subsequent myocardial infarctions. Similarly, individuals with heart failure and reduced left ventricular ejection fraction are likely to be unable to compensate for the increased circulatory demand induced by heat exposure. One might speculate that the observed association between heat exposure and increased respiratory disease mortality is also mediated by the underlying pathophysiological effect of heat exposure on right ventricular heart failure.

Unfortunately, climate change does not only change temperatures but also has negative effects on other environmental conditions, including air pollution, such as from the increased occurrence of wildfires. Fine particulate matter is causally implicated in cardiovascular disease morbidity and mortality ${ }^{8}$. Studies have impressively demonstrated the joint and severe effect of episodes of exposure to heat and particulate matter on cardiovascular disease mortality, for example in Russia in $2010^{\circ}$. A nationwide study in Italy showed that the effect of heat on mortality is tripled under conditions of high concentrations of fine particulate matter compared with conditions of low concentrations of fine particulate matter ${ }^{10}$.

Taken together, these data emphasize the need to act now. Mitigating climate change by achieving a 


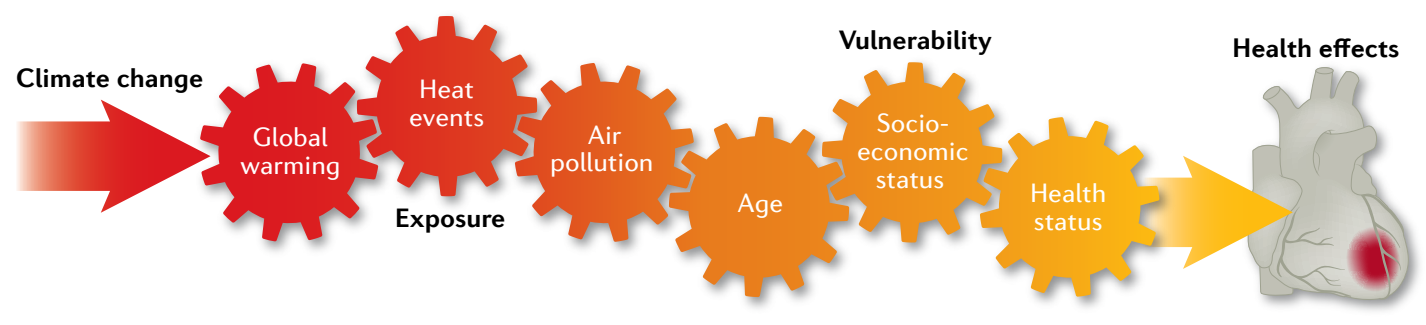

Fig. 1 | Factors contributing to the cardiovascular risks of climate change. Global warming is leading to more frequent extreme heat events. The health effects of the exposure to heat are further increased by air pollution. Older individuals, individuals with low socioeconomic status and those with underlying conditions associated with an increased risk of cardiovascular disease, such as type 2 diabetes mellitus and hypertension, are the most vulnerable to heat-related acute cardiovascular disease events, such as myocardial infarction.

zero-carbon emission economy is essential. For example, investments in a low-carbon public transport system and safe walking and cycling networks promote a switch from passive to active travel. These measures decrease greenhouse gas emissions and reduce air pollution, while also promoting physical activity and offering benefits for cardiovascular health. These much-needed political changes will reduce the cardiovascular disease burden for future generations, but we must also consider the immediate implications for preventing and treating cardiovascular diseases. Heat warning systems have been implemented in many countries, regions or cities. However, concerns persist about whether the most vulnerable groups, such as elderly or homeless individuals, are being adequately reached. The individual risks of the severe consequences of heat might only partially be addressed owing to underlying cardiovascular diseases and treatment regimens. Therefore, we must increase awareness and enable patients with cardiovascular diseases to take preventive measures. Likewise, physicians are encouraged to raise awareness and potentially to adjust treatment regimens. To support this effort, further research on health status monitoring via wearable devices and ambulatory measurements of blood pressure, arrhythmia or dysglycaemia and potential interactions with specific medications and accompanying comorbidities is needed. In particular, type 2 diabetes and respiratory diseases are highly relevant. In the ongoing coronavirus disease 2019 (COVID-19) pandemic, co-exposure to COVID-19 and heat might increase the burden of disease in affected individuals. Similarly, co-exposure to COVID-19 and cold might increase the risk of mortality beyond the obvious increased likelihood of SARS-CoV-2 infection during the winter months.

In conclusion, the combined effects of global warming, air pollution and also individual age, socioeconomic status and health status are expected to result in an increase in heat-related deaths within the coming decades (FIG. 1). Climate change already confers an increased risk of cardiovascular disease events in the general population, which emphasizes the urgency to implement policy measures to achieve the Paris Agreement. Even in the best-case scenario, these environmental changes are already resulting in quantifiable and avoidable acute cardiovascular disease events and should be considered as part of our efforts to prevent and treat cardiovascular diseases.

1. IPCC. Special Report: Global Warming of $1.5^{\circ} \mathrm{C}$ https://www.ipcc.ch/ sr15/ (2018).

2. Robine, J. M. et al. Death toll exceeded 70,000 in Europe during the summer of 2003. C. R. Biol. 331, 171-178 (2008).

3. Watts, N. et al. The 2019 report of The Lancet Countdown on health and climate change: ensuring that the health of a child born today is not defined by a changing climate. Lancet 394, 1836-1878 (2019).

4. GBD 2019 Risk Factor Collaborators. Global burden of 87 risk factors in 204 countries and territories, 1990-2019: a systematic analysis for the Global Burden of Disease Study 2019. Lancet 396 1223-1249 (2020).

5. Chen, K. et al. Temporal variations in the triggering of myocardial infarction by air temperature in Augsburg, Germany, 1987-2014. Eur. Heart J. 40, 1600-1608 (2019).

6. Chen, K. et al. Projection of temperature-related myocardial infarction in Augsburg, Germany: moving on from the Paris Agreement on climate change. Dtsch. Arztebl. Int. 116, 521-527 (2019).

7. Schneider, A., Ruckerl, R., Breitner, S., Wolf, K. \& Peters, A. Thermal control, weather, and aging. Curr. Environ. Health Rep. 4, 21-29 (2017)

8. Brook, R. D. et al. Particulate matter air pollution and cardiovascular disease: an update to the scientific statement from the American Heart Association. Circulation 121, 2331-2378 (2010).

9. Shaposhnikov, D. et al. Mortality related to air pollution with the Moscow heat wave and wildfire of 2010. Epidemiology 25, 359-364 (2014).

10. Scortichini, M. et al. Short-term effects of heat on mortality and effect modification by air pollution in 25 Italian cities. Int. J. Environ. Res. Public Health 15, 1771 (2018).

\section{Acknowledgements}

We thank M. Dallavalle (Helmholtz Center Munich, Germany) for help with designing the figure before submission and K. Chen (Yale University, New Haven, CT, USA) for discussions on the topic. This work was supported by the HERA project (No. 825417) and the Exhaustion project (No. 820655) funded by the European Union Horizon 2020 Program and the HI-CAM project funded by the Helmholtz Association.

\section{Competing interests}

The authors declare no competing interests. 